\title{
COMPARAÇÃO DA DIETA NATURAL DO SIRI-AZUL CALLINECTES SAPIDUS RATHBUN, 1896 (CRUSTACEA: DECAPODA: PORTUNIDAE) EM DOIS LOCAIS NO ESTUÁRIO DA LAGOA DOS PATOS, RS, BRASIL.
}

\author{
LEONARDO SIMÕES FERREIRA, ANDREIA BARROS, ROBERTA ARAUJO BARUTOT \& FERNANDO D'INCAO \\ Universidade Federal do Rio Grande (FURG) - Instituto de Oceanografia - Laboratório de Crustáceos Decápodes, Caixa-Postal: 474 - CEP \\ 96650-900 - Rio Grande - RS - Brasil. leocrab@bol.com.br
}

\section{RESUMO}

O siri-azul Callinectes sapidus Rathbun, 1896 é o mais abundante dentre as espécies do gênero na região estuarina da Lagoa dos Patos, sendo considerado um predador bentônico chave. O presente estudo tem por objetivo fazer a comparação da dieta natural do siri-azul entre dois locais do estuário da Lagoa dos Patos. As coletas foram realizadas em fevereiro e maio de 2009 com rede de arrasto de portas durante 15 minutos. No laboratório, os intestinos foram pesados e o grau de repleção avaliado. A contribuição de cada item alimentar foi analisada com a freqüência de ocorrência e a freqüência relativa dos pontos. Para comparação entre os dois pontos de coleta foi realizado teste $\mathrm{G}$ com fator de correção de Williams $(p<0,05)$. Nas Bandeirinhas foi possível identificar 18 itens, os mais ocorrentes foram resto vegetal, Erodona mactroides, Heleobia australis e Brachyura; no Saco da Mangueira foi possível identificar 12 itens, entre os mais ocorrentes estão resto vegetal, Erodona mactroides e camarão. Foi observada diferença entre os itens ocorrentes na dieta de acordo com o local de coleta. O siri-azul se caracteriza por ser generalista oportunista, diferindo sua alimentação de acordo com a disponibilidade de presas do ambiente.

PALAVRAS CHAVE: dieta alimentar, Callinectes, Lagoa dos Patos, siri azul

\section{ABSTRACT}

Comparison of natural diet of blue crab Callinectes sapidus Rathbun, 1896 (Crustacea: Decapoda: Portunidae) in two places at Patos Lagoon estuary, RS, Brazil

The blue crab Callinectes sapidus Rathbun, 1896 is the most abundant species of genus in the estuarine region of Patos Lagoon, considered as key benthic predator. This study aims to compare the natural diet of the blue crab between two sites in the estuary of Patos Lagoon. Samples were collected in February and May 2009 by using an otter trawl towed during 15 minutes. In the laboratory, the intestines were weighed and assessed the degree of fullness. The contribution of each food item was analyzed by using the frequency of occurrence and relative frequency of points. The comparison between both sites was performed using $G$ test with Williams correction factor $(p<0.05)$. At Bandeirinhas were identified in 18 items, the rest were more occurring plant Erodona mactroides, Heleobia australis and Brachyura. At Saco da Mangueira 12 items were identified, among the rest are occurring plant Erodona mactroides and shrimp. Difference was found among the items occurring in the diet according to the collection site. The blue crab is characterized by opportunistic generalist, its diet differs according to prey availability in the environment

KEYWORDS: diet, Callinectes, Patos Lagoon, blue crab.

\section{INTRODUÇÃO}

Callinectes sapidus Rathbun, 1896 é uma espécie de grande valor econômico, sendo explorado na costa Atlântica dos EUA e da América Central (Olmi \& Orth, 1995). No Brasil, capturas comerciais são realizadas no estado de Santa Catarina enquanto que no Rio Grande do Sul as capturas ocorrem como subproduto da pesca artesanal de peixes e camarões (Ferreira \& D'Incao, 2008). A espécie é considerada ainda como o braquiúro mais importante, do ponto de vista pesqueiro, da região estuarina da Lagoa dos Patos, RS (Ceperg/lbama, 2002).

O siri azul é classificado como predador bentônico chave por controlar a abundância de outras espécies bentônicas estuarinas, consumindo uma grande variedade de organismos tanto da infauna como epifauna (Hines et al., 1990). Por serem vorazes predadores, os braquiúros podem exercer importantes efeitos sobre a estrutura e função de sistemas aquáticos e afetar diretamente a abundância e a estrutura de tamanhos de suas presas, através da remoção selecionada de certos indivíduos da comunidade predada (Wright et al., 1996).

A alimentação da espécie já foi foco de alguns trabalhos como os de Laughlin (1982) que realizou estudos em Apalachicola Bay, Flórida (EUA); Kapusta \& Bemvenuti (1998) investigaram a atividade nictemeral de alimentação de $C$. sapidus numa pradaria de Ruppia maritima numa enseada estuarina da Lagoa dos Patos; Oliveira et al. (2006) analisou a dieta na região estuarina da Lagoa dos Patos em relação as variações sazonais. Para outras espécies do gênero Callinectes, Branco \& Verani (1997) realizaram estudo sobre a alimentação de $C$. danae na Lagoa da Conceição / SC, e Mantelatto \& Christofoletti (2001) analisaram a dieta de C. ornatus na Baia de Ubatuba / SP.

Diferentemente de Oliveira et al. (2006) que considerou o estuário da Lagoa dos Patos como um todo; esse manuscrito considera a possível diferença na dieta dessa espécie entre as regiões do Saco da 
Mangueira e o sul da llha dos Marinheiros Bandeirinhas, contribuindo assim com novas

\section{MATERIAIS E MÉTODOS}

\section{Área de estudo}

As coletas foram realizadas em dois pontos do estuário da Lagoa dos Patos, RS, Brasil, no sul da Ilha dos Marinheiros, mais precisamente no local chamado "Bandeirinhas" (32 $\left.02^{\circ} \mathrm{S} 052^{\circ} 12 \mathrm{~W}\right)$ e no Saco da Mangueira $\left(32^{\circ} 05^{\prime} \mathrm{S} 052^{\circ}\right.$ 08W) (Figura 1).

O Saco da Mangueira é uma enseada rasa, semi fechada, de forma ovalada e localiza-se ao sul da informações sobre a dieta dessa espécie.

cidade do Rio Grande, possui $23 \mathrm{Km}^{2}$ de área submersa e uma profundidade máxima de 1,5m. Funciona como um pequeno estuário dentro do maior (Lagoa dos Patos), onde desemboca. Nessa enseada destaca-se o lançamento do principal efluente oficial doméstico da cidade (sem tratamento) nas proximidades da entrada da enseada, além dos lançamentos de vários efluentes domésticos clandestinos e de efluentes de indústrias marginais com atividades bastante diversificadas (Almeida et al., 1993).

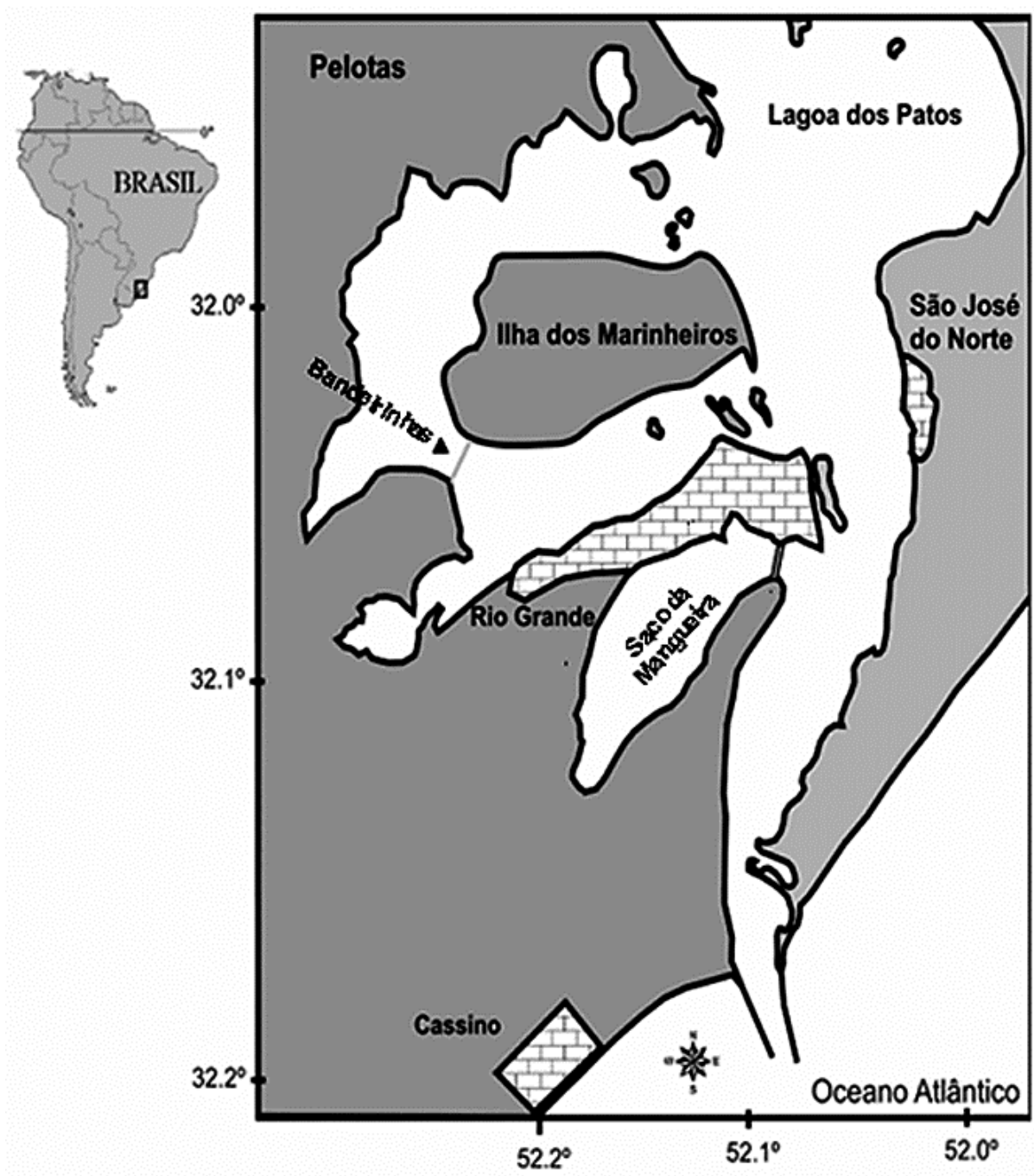

FIGURA 1 - Lagoa dos Patos e região adjacente com as áreas de amostragem de Callinectes sapidus Rathbun, 1896

A llha dos Marinheiros localiza- se a margem oeste do estuário no município de Rio Grande é a maior ilha do complexo estuarino da Lagoa dos Patos abrangendo uma área de aproximadamente $62 \mathrm{Km}^{2}$, sendo caracterizada por campos alagadiços, lagos rasos intermitentes, cordões de dunas e mantos arenosos. Pequenas formações de mata arenosa e mata palustre estão presentes em alguns pontos (Vieira, 1983). 


\section{Metodologia de coleta}

Foram realizadas duas coletas durante os meses de fevereiro e maio (verão/outono) de 2009. Os animais foram coletados no período diurno com uma rede de arrasto de portas por um período de 15 minutos. O tamanho das malhas medidas entre nós opostos variavam entre 76 e $78 \mathrm{~mm}$ no corpo da rede e entre 60 e $66 \mathrm{~mm}$ no saco, o que caracteriza a captura dos indivíduos adultos.

Após a coleta os animais foram acondicionados em um balde com formol $10 \%$, por um período de vinte e quatro horas para retardar o processo de digestão. Após, os animais foram separados por sexo e feita a biometria (medida da largura da carapaça em centímetros) com auxílio de paquímetro e a análise dos conteúdos alimentares. A largura da carapaça (LC) foi considerada como a distância entre os últimos espinhos ânterolaterais. Cada animal foi pesado em gramas (exatidão 0,01g). Estes procedimentos foram realizados no Laboratório de Crustáceos Decápodos Instituto de Oceanografia FURG.

Após a biometria os animais foram dissecados, o intestino foi retirado, pesado, avaliado visualmente em relação ao grau de repleção (quantidade de alimento presente no seu interior) e classificado de acordo com Santos (1978) nas seguintes classes: classe $3(100 \%-70,1 \%)$; classe $2(70 \%-30,1 \%)$; classe $1(30 \%-0,1 \%)$; classe $0(0 \%)$. O conteúdo, devidamente identificado, foi depositado em placa de Petri e com o auxílio de um microscópio estereoscópico.

Os itens alimentares foram identificados ao menor nível taxonômico possível, os que não puderam ser identificados, devido ao seu elevado grau de digestão foram considerados como matéria orgânica indeterminada (MOI), os restos de origem animal que não puderam ser identificados com uma maior exatidão foram considerados como resto animal, a vegetação não identificada foi considerada como resto vegetal e a areia foi considerada como sedimento. O índice de repleção médio (Ir) foi estimado de acordo com Santos (1978) e relaciona o peso do animal com o peso do intestino, assim podese avaliar se o animal consumiu mais ou menos presas.

A contribuição relativa de cada item, no volume total do conteúdo do intestino, foi mensurada segundo o método dos pontos proposto por Williams (1981), sendo subjetivamente atribuída uma escala de pontos com cinco categorias. $O$ item recebeu 100 pontos apresentando valores de volume entre $100 \%$ e $95,1 \%$ do conteúdo do intestino; 75 pontos entre 95\% e $65,1 \%$; 50 pontos entre $65 \%$ e $35,1 \%$; 25 pontos entre $35 \%$ e $5,1 \%$; e 2,5 pontos para volumes com valores menores que $5 \%$. O número de pontos recebidos por cada item foi relativo ao grau de repleção do intestino no qual se encontrava. Esta atribuição consiste em multiplicar o número de pontos por um valor dependente das classes de repleção: classe $3-1,00$; classe 2 - 0,60; classe $1-0,20$, (classe $3=$ número de pontos $\times 1,00$; classe $2=$ número de pontos $\times 0,60$; classe $1=$ número de pontos $\times 0,20)$. Assim, 0 máximo de pontos que um simples item em um único intestino pode obter é $100(100 \times 1,00$, onde está presente somente um item em um intestino completamente cheio). O mínimo possível é 0,5 (2,5 x 0,20 , onde 0 item representa menos de $5 \%$ de um intestino quase vazio). Os intestinos da classe 0 foram desconsiderados por não possuírem nenhum item alimentar em seu interior. A porcentagem para cada item foi calculada segundo a fórmula: $\mathrm{FP}=\Sigma \mathrm{jn}=\mathrm{i}(\mathrm{PFaij} / \mathrm{A}) \times 100$, onde $a_{i j}$ é o número de pontos que cada item $i$ obteve no intestino $j$ de cada animal e $A$ é o número total de pontos para todos os itens em todos os animais da amostra.

A freqüência de ocorrência foi calculada segundo a fórmula: $\mathrm{FO}=b_{i} / \mathrm{N} \times 100$, onde $b_{i}$ é o número de animais nos quais o item $i$ está presente e $N$, o número de animais da amostra (todos os que possuem conteúdo no intestino).

Para fazer a comparação da dieta natural de $C$. sapidus entre dois pontos do estuário da Lagoa dos Patos foi utilizado o teste $\mathrm{G}$ com o fator de correção de Williams, através do programa BioEstat $5.0 \mathrm{com}$ nível de significância de 0,05. 


\section{RESULTADOS}

Foram analisados 107 intestinos de C. sapidus, sendo 57 do local Bandeirinhas, no sul da llha dos Marinheiros (28 machos e 29 fêmeas) e 50 do Saco da Mangueira (23 machos e 27 fêmeas).

Dentre os 57 intestinos analisados nas Bandeirinhas, 9,7\% dos intestinos dos machos estavam vazios e $90,4 \%$ apresentaram conteúdo, das fêmeas analisadas $100 \%$ dos intestinos tinham algum tipo de alimento. Dos 50 intestinos analisados dos animais capturados no Saco da Mangueira, 11,5\% dos intestinos dos machos estavam vazios e $88,5 \%$ apresentavam algum tipo de item alimentar, das fêmeas analisadas $7,1 \%$ dos intestinos estavam vazios e $92.8 \%$ com alimento (Figura 2 ).

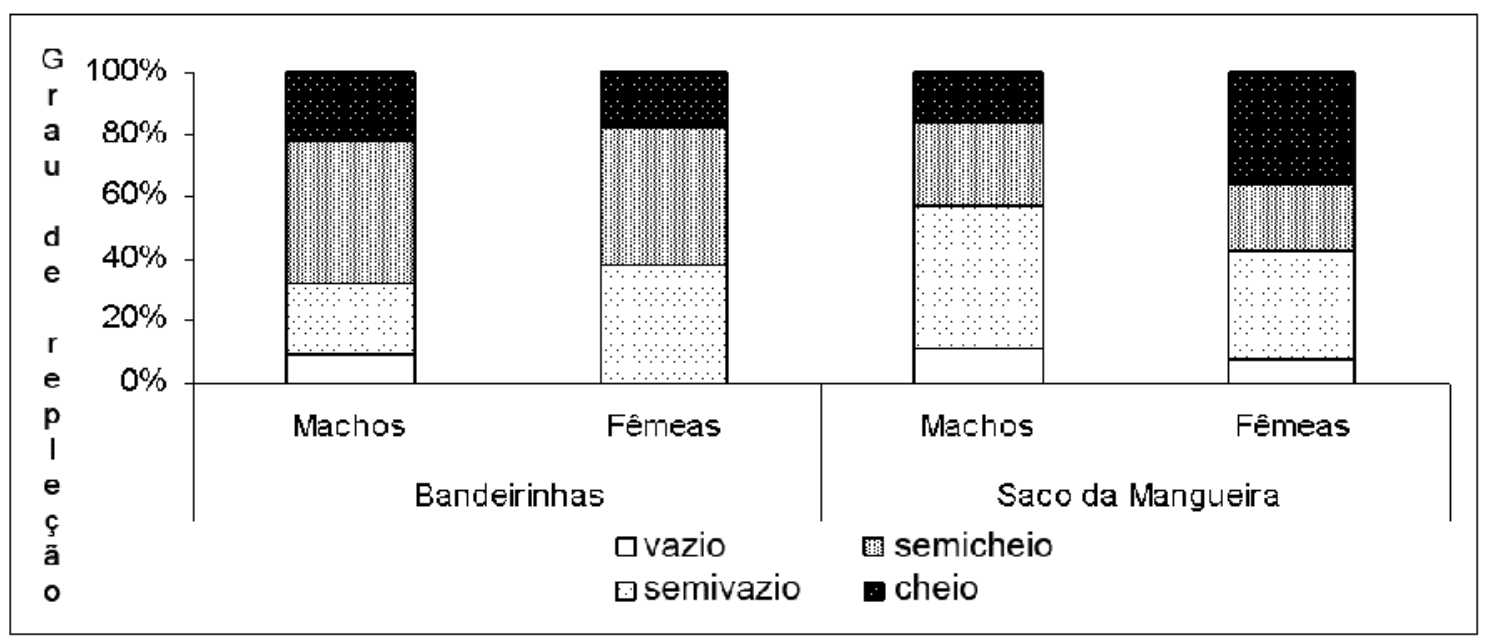

FIGURA 2 - Grau de Repleção dos intestinos anteriores de machos e fêmeas de Callinectes sapidus para os dois locais de coleta.

O índice de repleção médio (Ir) de C. sapidus coletados nas Bandeirinhas foi 0.016 para os machos e 0.015 para as fêmeas. No Saco da Mangueira foi de
0.016 para os machos e 0.017 para as fêmeas (Figura 3).

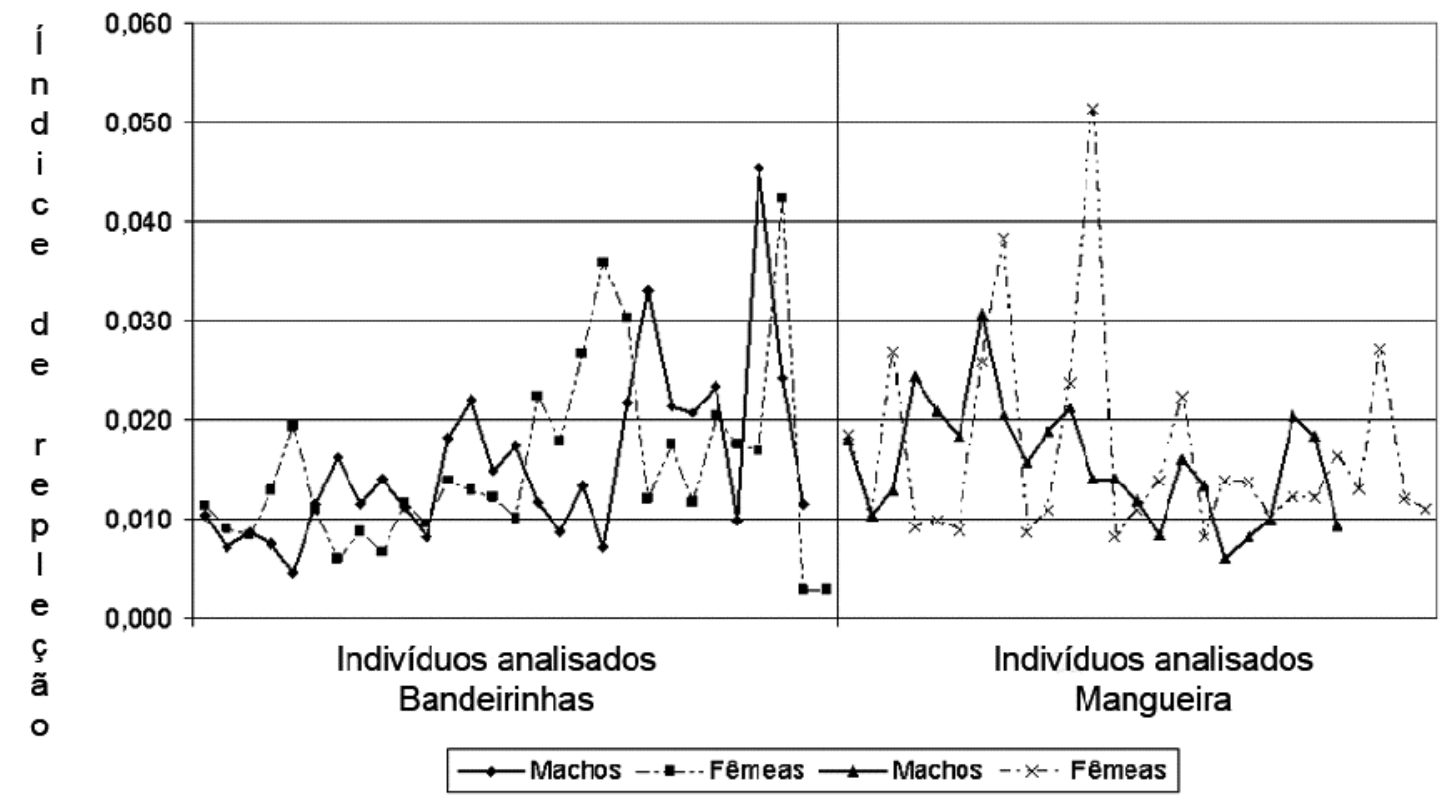

FIGURA 3 - Índice de Repleção Médio dos intestinos anteriores de machos e fêmeas de Callinectes sapidus para os dois locais de coleta. 
Nos exemplares coletados nas Bandeirinhas foi possível identificar 18 itens componentes da dieta. Os itens com maior frequência de ocorrência foram restos vegetais, moluscos (Erodona mactroides e Heleobia australis) e brachyura. Os de menor ocorrência foram Oligochaeta, Insecta, Mysida e sementes de Ruppia sp. No Saco da Mangueira foram identificados 12 itens alimentares, sendo os de maior frequência de ocorrência os restos vegetais, moluscos (Erodona mactroides e Heleobia australis) e camarão-rosa (Farfantepenaeus sp.). Os de menor ocorrência foram os Ostracoda e restos animais (Tabela 1).

TABELA 1 - Freqüência Relativa dos pontos (P\%) e Freqüência de Ocorrência $(O \%)$ dos itens alimentares presentes nos intestinos anteriores de machos e fêmeas de Callinectes sapidus coletados nas Bandeirinhas e no Saco da Mangueira.

\begin{tabular}{|c|cc|cc|}
\hline Itens Alimentares & \multicolumn{2}{|c|}{ Bandeirinhas } & \multicolumn{2}{c|}{ Saco da Mangueira } \\
\hline & $\mathrm{P} \%$ & $0 \%$ & $\mathrm{P} \%$ & $0 \%$ \\
\hline Semente de Ruppia sp. & 0.1 & 1.8 & 0 & 68.0 \\
Resto vegetal & 12.0 & 77.2 & 11.5 & 30.0 \\
Heleobia australis & 6.1 & 42.1 & 4.3 & 40.0 \\
Erodona mactroides & 33.0 & 52.6 & 11.5 & 38.0 \\
Brachyura & 8.3 & 35.1 & 14.9 & 40.0 \\
Camarão & 2.3 & 7.0 & 13.9 & 4.0 \\
Ostracoda & 1.5 & 10.5 & 1.4 & 10.0 \\
Cirripedia & 0 & 0 & 0.6 & 0 \\
Amphipoda & 2.0 & 3.5 & 0 & 0 \\
Isopoda & 1.1 & 3.5 & 0 & 0 \\
Mysida & 0.1 & 1.8 & 0 & 0 \\
Neanthes succinea & 1.3 & 5.3 & 0 & 10.0 \\
Laeonereis acuta & 2.0 & 3.5 & 0.5 & 26.0 \\
Peixe & 3.2 & 12.3 & 9.6 & 0 \\
Oligochaeta & 0.1 & 1.8 & 0 & 0 \\
Insecta & 0.1 & 1.8 & 0 & 36.0 \\
Sedimento & 19.2 & 75.4 & 10.2 & 8.0 \\
Resto animal & 0.8 & 14.0 & 1.7 & 36.0 \\
Matéria orgânica & 6.9 & 24.6 & 19.9 & \\
Indeterminada & & & & 0 \\
\hline
\end{tabular}

O teste $\mathrm{G}$ com o fator de correção de Williams $(p<0,05)$ aplicado a frequência absoluta de ocorrência dos 19 itens alimentares para os dois locais de captura não apresentou diferença significativa na proporção dos itens alimentares consumidos por machos e fêmeas $(p=0.5684$ - Bandeirinhas e $p=$ 0.3811 - Saco da Mangueira). O resultado do teste $G$ agrupando-se os sexos e comparando-se os dois locais obteve-se uma diferença significativa na dieta dos animais $(p=0,0004)$. 


\section{DISCUSSÃO}

A frequência de indivíduos com intestino anterior vazio foi acentuadamente mais baixa do que aqueles com conteúdo alimentar em ambos os sexos e ambos os locais de captura. Oliveira et al. (2006) em seu trabalho de análise da dieta do siri-azul na região estuarina da Lagoa dos Patos, também observaram que aproximadamente $90 \%$ dos intestinos anteriores de C. sapidus apresentaram algum tipo de conteúdo alimentar. Os animais capturados nas Bandeirinhas apresentaram menor proporção de intestinos vazios comparando com a dos animais do Saco da Mangueira, talvez devido a uma maior diversidade de presas encontrada neste local.

Nos dois locais as fêmeas apresentaram uma porcentagem menor de intestinos vazios que os machos. Oliveira et al. (2006) observaram uma maior proporção de intestinos vazios para as fêmeas do que para machos. Diferenças de repleção de intestinos de machos e fêmeas de $C$. sapidus também puderam ser observadas por Laughlin (1982), no estuário de Apalachicola, Flórida (EUA).

A alta porcentagem de intestinos anteriores de C. sapidus com alimento indica uma alta taxa de predação/alimentação desta espécie de acordo com Oliveira et al. (2006). Edgar (1990) afirma que os portunídeos apresentam maior atividade $e$ crescimento que a maioria dos crustáceos, podendo ser esta a explicação para a constante voracidade relatada em estudos alimentares com espécies dessa família.

Comparando-se os dois locais de coleta, observa-se uma maior variedade de itens alimentares nas Bandeirinhas a qual apresentou exclusividade para alguns itens nessa região: semente de Ruppia sp., Amphipoda, Isopoda, Mysida, N. succinea, Oligochaeta e Insecta. Já o item Cirripedia apareceu na dieta dos animais somente do Saco da Mangueira. Kapusta \& Bemvenuti (1998), estudando a atividade nictemeral da alimentação de juvenis de $C$. sapidus numa pradaria e num plano não-vegetado, observaram a dominância de itens alimentares de acordo com os distintos habitats.

Os moluscos $E$. mactroides e $H$. australis obtiveram índices significativos de ocorrência em ambos locais estudados, corroborando com o trabalho de Oliveira et al. (2006) que apresentou também um alto índice de moluscos predados, sendo este o grupo mais ocorrente em seus estudos. Kapusta \& Bemvenuti (1998), verificaram maior freqüência do gastrópode $H$. australis para os juvenis das enseadas rasas do estuário da Lagoa dos Patos. A presença de moluscos na dieta dos braquiúros, em especial portunídeos, é de grande importância e foi registrada por vários autores (Laughlin, 1982; Hines et al., 1990; Mantelatto \& Christofoletti, 2001).

A alta freqüência de ocorrência de resto vegetal no conteúdo alimentar de $C$. sapidus pode ser visto também no trabalho Oliveira et al. (2006) sendo que a constante presença do item resto vegetal no conteúdo alimentar do siri-azul caracteriza a espécie como generalista, já que também possui hábitos claramente carnívoros. Mantelatto \& Christofoletti (2001) não consideraram resto vegetal entre os quatro itens de maior importância da dieta natural de Callinectes ornatus. Branco \& Verani (1997) também reportaram material de origem vegetal no estômago de $C$. danae na Lagoa da Conceição / SC, porém esta espécie foi considerada como exclusivamente carnívora, já que a presença do material vegetal foi rara. Kapusta \& Bemvenuti (1998) também observaram filamentos e sementes de macrófitas em juvenis de $C$. sapidus. No presente estudo, o item Resto Vegetal teve uma alta freqüência de ocorrência devido ao fato do estuário da Lagoa dos Patos ser formado por cerca de 170 $\mathrm{km}^{2}$ de áreas rasas $(<1.5 \mathrm{~m})$, as quais fornecem condições propicias ao estabelecimento $\mathrm{e}$ desenvolvimento de fanerógamas submersas enraizadas e macroalgas bentônicas (Copertino \& Seeliger, 2010). Esse fator corrobora com há maior ocorrência de camarão-rosa no Saco da Mangueira, devido ao fato dessa região ser considerada como um berçário para o desenvolvimento de diversas espécies de peixes e crustáceos (D’Incao, 1991).

$O$ item sedimento foi um dos itens com freqüência relativa dos pontos e freqüência de ocorrência intermediária. No presente estudo esta presença pode ser atribuída à ingestão acidental, como sugere o trabalho de Branco \& Verani (1997). Porém, uma parte desta ingestão pode ser atribuída 
como alimento propriamente dito, devido à grande quantidade de matéria orgânica associada ao sedimento (D'Incao et al., 1990; Barutot et al., 2011). Williams (1982) atribui este fator a uma ingestão proposital, utilizada como fonte de minerais para a formação de uma nova carapaça. A ingestão pode estar também auxiliando em processos digestórios, através do contato mecânico, para a trituração de presas calcárias, como gastrópodes e bivalves (Mantelatto \& Christofoletti, 2001).

No presente estudo a contribuição de decápodes foi bastante elevada em relação aos outros crustáceos ocorrentes, sua presença já era esperada e pode ser explicada pelo fato da existência de canibalismo e predação interespecífica, em condições ambientais, entre os braquiúros, principalmente sobre juvenis, indivíduos em ecdise ou doentes (Williams, 1982; D'Incao et al., 1990; Barutot et al., 2011). Trabalhos com outros decápodes têm relatado que a ingestão da própria exúvia é uma das principais maneiras para a reposição de cálcio (Hartnoll, 1982).

De acordo com as análises feitas na dieta alimentar de $C$. sapidus nos dois pontos do estuário da Lagoa dos Patos, pode-se dizer que resto vegetal, moluscos e sedimento, são itens de maior importância na alimentação do siri-azul, pois estes aparecem entre os primeiros tanto na frequência de ocorrência como na frequência relativa dos pontos, em ambos locais, os itens secundários são crustáceos de vários tipos de acordo com a disponibilidade destes no ambiente onde estão inseridos. Portanto sugere-se que $C$. sapidus se caracteriza por ser generalista oportunista, diferindo sua alimentação de acordo com a disponibilidade do ambiente.

\section{AGRADECIMENTOS}

A todos que contribuíram para a realização deste trabalho. Aos pescadores do estuário da Lagoa dos Patos. A estagiária Andréia por todo seu empenho no decorrer deste. $E$ ao apoio financeiro da bolsa concedido pela CAPES.

\section{REFERÊNCIAS}

ALMEIDA, MT, MGZ BAUMGARTEN \& RMS RODRIGUES. 1993. Identificação das possíveis fontes de contaminação das águas que margeiam a Cidade de Rio Grande, RS. Série Documentos Técnicos 06 - Oceanografia. Ed. FURG. Rio Grande. 36p.

BARUTOT, RA, F D'INCAO \& DB FONSECA. 2011. Natural Diet of Neohelice granulata (Dana, 1851) (Crustacea, Varunidae) in Two Salt Marshes of the Estuarine Region of the Lagoa dos Patos Lagoon. Braz. Arch. Biol. Technol. 54 (1): 91-98.

BRANCO, JO \& JR VERANI. 1997. Dinâmica da alimentação natural de Callinectes danae Smith (Decapoda, Portunidae) na Lagoa da Conceição, Florianópolis, Santa Catarina, Brasil. Rev. Bras. Zool., 14 (4): 1003-1018.

CEPERG/IBAMA. 2002. Desembarque de pescados no Rio Grande do Sul. Porto Alegre, Instituto Brasileiro do Meio Ambiente e dos Recursos Naturais Renováveis. sem paginação.

COPERTINO, M. \& SEELIGER, U. 2010. Hábitats de Ruppia maritima e de macroalgas. In: SEELIGER, UC \& ODEBRECHT, C. (eds.), O estuário da Lagoa dos Patos. Um século de transformações. Ed. FURG, Rio Grande-RS, 91-98.

D'INCAO, F, KG SILVA, ML RUFINO, \& AC BRAGA. 1990. Hábito alimentar do caranguejo Chasmagnathus granulata Dana, 1851 na Barra do Rio Grande, RS (Decapoda, Grapsidae). Atlântica, 12 (2): 85-93.

D'INCAO, F. 1991. Pesca e biologia de Penaeus paulensis na Lagoa dos Patos, RS. Atlântica, Rio Grande, 13 (1): 159-169.

EDGAR, GJ. 1990. Predator-prey interactions in seagrass beds. II. Distribution and diet of the blue manna crab Portunus pelagicus Linnaeus at Cliff Head, Western Australia. J. Exp. Mar. Biol. Ecol., 139 (1-2): 23-32.

FERREIRA, LS \& F D'Incao. 2008. Crescimento de Callinectes sapidus (Crustacea, Decapoda, Portunidae) no estuário da laguna dos Patos, RS, Brasil. Iheringia, Sér. Zool., 98 (1): 70-77.

HARTNOLL, RG 1982. Growth. In: Lawrence G. Abele (ed.) The biology of Crustacea. Embriology, morphology and genetics. Academic Press, (2): 111- 196.

HINES, AH, AM HADDON \& LA WIECHERT. 1990. Guild structure and foraging impact of blue crabs and epibenthic fish in a subestuary of Chesapeake Bay. Mar. Ecol. Prog. Ser., 67 (2): 105-126.

KAPUSTA, SC \& CE BEMVENUTI. 1998. Atividade nictemeral de alimentação de juvenis de Callinectes sapidus Rathbun, 1896 (Decapoda: Portunidae) numa pradaria de Ruppia maritima e num plano não vegetado, numa enseada estuarina da Lagoa dos Patos, RS, Brasil. Nauplius, 6: 41-52.

LAUGHLIN, RA. 1982. Feeding habitats of the blue crab, Callinectes sapidus Rathbun, in the Apalachicola estuary, Florida. Bull. Mar. Sci., 32: 807-822.

MANTELATTO, FLM \& RA CHRISTOFOLETTI. 2001. Natural feeding activity of the crab Callinectes ornatus (Portunidae) in Ubatuba Bay (São Paulo, Brazil): influence of season, sex, size and molt stage. Mar. Biol., 138 (3): 585-594.

OLIVEIRA, A, TK PINTO, DPD SANTOS \& F D'INCAO. 2006. Dieta natural do siri-azul Callinectes sapidus (Decapoda,Portunidae) na região estuarina da Lagoa dos Patos,Rio Grande, Rio Grande do Sul, Brasil, Iheringia, Sér. Zool., 96(3): 305-313.

OLMI, EJ \& RJ ORTH. 1995. Introduction to the Proceedings of the Blue Crab Recruitment Symposium. Bull. Mar. Sci. 57 (3): 707-716.

SANTOS, EP. 1978. Dinâmica de populações aplicada a pesca e piscicultura. São Paulo, HUCITEC/EDUSP. 129p. 
VIEIRA, EF. 1983. Rio Grande: geografia física, humana e econômica. Porto Alegre, Sagra. $158 \mathrm{p}$.

WILLIAMS, MJ. 1981. Methods for analysis of natural diet in portunid crabs (Crustacea: Decapoda: Portunidae). J. Exp. Mar. Biol. Ecol., 52(1): 103-113.

WILLIAMS, MJ. 1982. Natural food and feeding in the commercial sand crab Portunus pelagicus Linnaeus, 1766 (Crustacea:
Decapoda: Portunidae) in Moreton Bay, Queensland. J. Exp. Mar. Biol. Ecol., 59(2-3): 165-176.

WRIGHT, RA, LB CROWDER, \& TH MARTIN. 1996. Selective predation by blue crabs on the gastropod, Bittium varium: confirmation from opercula found in the sediments. Estuaries 19(1):75-81.

Submetido $-04 / 11 / 10$

Aceito $-23 / 10 / 11$ 